\title{
Pulse-modulated second harmonic imaging microscope quantitatively demonstrates marked increase of collagen in tumor after chemotherapy
}

\author{
Anju M. Raja \\ A $^{*}$ STAR \\ Institute of Bioengineering and Nanotechnology \\ Singapore 138669, Singapore \\ and \\ National University of Singapore \\ NUS Graduate School for Integrative Sciences \\ and Engineering \\ NUS Graduate Programme in Bioengineering \\ Singapore 117456, Singapore \\ and \\ National University Health System \\ Yong Loo Lin School of Medicine \\ Department of Medicine \\ Singapore 119228, Singapore
}

\section{Shuoyu Xu}

Singapore-MIT Alliance

E4-04-10

4 Engineering Drive 3

Singapore 117576, Singapore

and

Institute of Bioengineering and Nanotechnology

Singapore 138669, Singapore

\section{Wanxin Sun}

A STAR

Institute of Bioengineering and Nanotechnology

Singapore 138669, Singapore

\section{Jianbiao Zhou}

National University Health System

Yong Loo Lin School of Medicine

Department of Medicine

Singapore 119228, Singapore

and

National University of Singapore

Cancer Science Institute of Singapore

28 Medical Drive

Singapore 117597, Singapore

\section{Dean C. S. Tai}

Institute of Bioengineering and Nanotechnology

Singapore 138669 , Singapore

\section{Chien-Shing Chen}

National University Health System

Yong Loo Lin School of Medicine

Department of Medicine

Singapore 119228, Singapore

$$
\text { and }
$$

Loma Linda University

School of Medicine

Division of Hematology and Oncology

Loma Linda, California 92354

\author{
Jagath C. Rajapakse \\ Singapore-MIT Alliance \\ E4-04-10 \\ 4 Engineering Drive 3 \\ Singapore 117576, Singapore \\ and \\ Nanyang Technological University \\ School of Computer Engineering \\ Bioinformatics Research Center \\ Singapore 639798, Singapore
}

Peter T. C. So

Singapore-MIT Alliance

E4-04-10

4 Engineering Drive 3

Singapore 117576, Singapore

and

Singapore-MIT Alliance for Research \& Technology

S16-05-08

3 Science Drive 2

Singapore 117543, Singapore

and

Massachusetts Institute of Technology

Department of Mechanical Engineering

Cambridge, Massachusetts 02139

\section{Hanry Yu}

Institute of Bioengineering and Nanotechnology

Singapore 138669, Singapore

and

National University of Singapore

NUS Graduate School for Integrative Sciences and Engineering

NUS Graduate Programme in Bioengineering

Singapore 117456, Singapore and

Singapore-MIT Alliance

E4-04-10

4 Engineering Drive 3

Singapore 117576, Singapore and

Singapore-MIT Alliance for Research \& Technology

S16-05-08

3 Science Drive 2

Singapore 117543, Singapore and

Massachusetts Institute of Technology

Department of Mechanical Engineering

Cambridge, Massachusetts 02139

and

National University Health System

Yong Loo Lin School of Medicine

Department of Physiology

Singapore 117597, Singapore 
Abstract. Pulse-modulated second harmonic imaging microscopes (PM-SHIMs) exhibit improved signal-to-noise ratio (SNR) over conventional SHIMs on sensitive imaging and quantification of weak collagen signals inside tissues. We quantify the spatial distribution of sparse collagen inside a xenograft model of human acute myeloid leukemia (AML) tumor specimens treated with a new drug against receptor tyrosine kinase (ABT-869), and observe a significant increase in collagen area percentage, collagen fiber length, fiber width, and fiber number after chemotherapy. This finding reveals new insights into tumor responses to chemotherapy and suggests caution in developing new drugs and therapeutic regimens against cancers. () 2010 Society of Photo-Optical Instrumentation Engineers. [DOI: 10.1117/1.3497565]

Keywords: pulse modulation; second harmonic generation imaging; collagen modulation postchemotherapy; drug resistance.

Paper 10257R received May 12, 2010; revised manuscript received Aug. 5, 2010; accepted for publication Aug. 27, 2010; published online Oct. 14, 2010

\section{Introduction}

Drug development involves testing drug efficacy in animal models before clinical trials. It is important that the animal models of human diseases accurately recapitulate the pathophysiology and drug responses in the human body. It is also very important that the drug efficacy measurements are sensitive spatially and temporally. We have previously developed a xenograft model of human acute myeloid leukemia (AML) in severe combined immunodeficiency (SCID) mice and tested the efficacy of a newly developed compound (ABT-869) against tyrosine kinase receptors that demonstrated efficacy in reducing tumor size. ${ }^{1}$ An important issue that arises is whether the drug efficacy is sustained throughout the course of treatment to ensure the eventual elimination of last traces of cancer cells with suitable regimens or combination therapy.

As early 1986, Dvorak proposed that human tumors were actual "...wounds that do not heal.", Thus, using chemotherapy to treat cancer could induce a wound-healing response. Early effusion by histamine and enhancement of collagen biosynthesis contribute to the wound-healing process. Indeed, this phenomenon has been well documented in clinical observations. One main side effect of Bleomycin, an antitumor antibiotic, is lung fibrosis caused by enhanced production and deposition of collagen. Mitomycin C, an alkylating agent, induces an aberrant wound-healing response. ${ }^{3}$ Furthermore, the study of Tsuchiya et al. reveals that type- 5 collagen is significantly increased on the surface of bone in osteosarcoma patients after treatment with two commonly used chemodrugs: cisplatin and methotrexate. ${ }^{4}$ These findings suggest it is very likely that tumor cells are directly stimulated by chemotherapy to produce collagen. More recently, circulating collagen 4 has been identified as a biomarker of drug resistance to antiangiogenic therapy. ${ }^{5}$ Taken together, this evidence suggests that it is possible that the local wound-healing responses to anticancer drug treatment can impede further drug efficacy. We hypothesize that quantifying the spatial distribution of collagen inside tumors will yield a highly sensitive means to directly monitor the drug-induced barriers to further efficacy, and become a powerful tool to aid in the development of drug combinations or regimens that remain effica- cious to achieve complete tumor cell elimination. Understanding the process of collagen remodeling inside tumors will require more sophisticated tools for quantification.

In pursuing a quantitative tool to study the impact of chemotherapy in collagen production, we optically quantify the collagen before and after chemotherapy in tumors to investigate whether chemotherapy would induce collagen build-up inside tumors, which can impede drug delivery in subsequent chemotherapy. Collagen can be optically quantified in tissues by second harmonic generation (SHG) imaging, which visualizes noncentrosymmetric biomolecules such as myosin, and collagen types 1 and $4 .^{6,7}$ SHG imaging is especially suitable for tumor pathology studies, as there are no staining processes involved and thin tissue sections are not required, making sample preparation simple. ${ }^{8,9}$ The pulsed light source delivers high intensity laser pulses to the sample in femtosecond bursts with lower average power, reducing tissue damage. It is a quantitative technique, as it is not affected by dye concentrations or photobleaching problems. ${ }^{10}$ Since SHG is a secondorder phenomenon, the excitation signal can be confined in a small interrogation volume, reducing photodamage. ${ }^{11}$ The SHG imaging system has increased penetration depth compared to microscopes using lasers in the visible range, as the near-infrared source used in SHG systems is able to propagate deeper into the tissue, ${ }^{12,13}$ which is useful for studies in vitro or in vivo. ${ }^{14-18}$ Though we are not performing deep tissue imaging, the increased penetration depth can be exploited in future endoscopic and in-vivo imaging. SHG imaging of collagen in cancer models has been qualitatively demonstrated in melanoma, breast cancer, cervical, and ovarian cancer in animal and human studies. ${ }^{19-23}$ In the case of melanoma, ${ }^{24}$ the skin is a relatively accessible organ with a rich supply of collagen, and hence backward SHG of collagen fibers up to the depth of the melanoma capsule is possible; but within the actual tumor mass in melanoma, collagen fibers are reported to be sparse. ${ }^{25}$ In the case of solid tumors deep inside the host tissue, some studies focused on the stromal regions surrounding the tumor, ${ }^{26,27}$ while others who imaged the regions within the tumor qualitatively observed disruption in collagen fibers and loss of fine fibrils. ${ }^{23,28-30}$ Quantification of the fibers in the tumor interior have been reported to be difficult. ${ }^{31}$ Therefore, we have improved the conventional SHG imaging microscope (conventional SHIM) with a prism-based pulse modulator to quantify collagen with a pulse-modulated second harmonic imaging microscope (PM-SHIM) to visualize the fine fibrils within the tumor, and be able to quantify the collagen changes after drug administration to a xenograft model of human cancer.

The positive chirp or dispersion introduced by the optical components in the imaging system introduce group velocity dispersion (GVD), where the longer wavelength light in the pulse travels faster than the shorter wavelength light. ${ }^{32,33}$ This results in pulse broadening and reduction in peak power. SHG depends directly on the peak power delivered to the sample, thus GVD reduces the SHG susceptibility of the sample. Pulse modulators can be introduced in the optical path of the microscope to introduce negative chirp that counteracts the positive chirp introduced by dispersive optical components. ${ }^{34}$ SHG signals have been pulse modulated to improve signal-to-noise ratio (SNR) in qualitatively imaging human skin and mouse kidney. ${ }^{35,36}$ We have demonstrated that the increased sensitiv- 

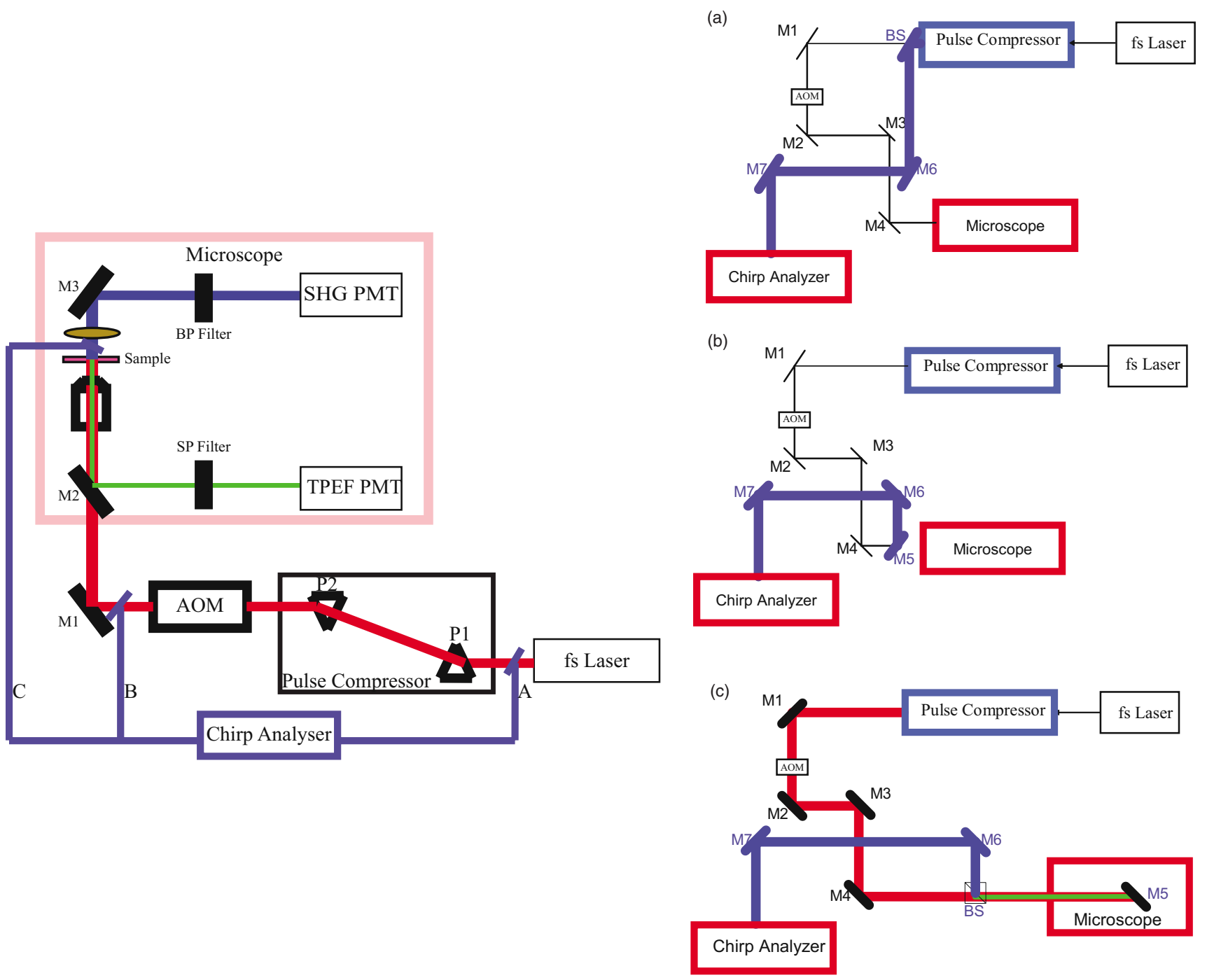

Fig. 1 Schematic of the PM-SHIM setup. The setup of the PM-SHIM is shown on the left and the alignment configuration for chirp analysis is shown in the right. The PM-SHIM consists of a femtosecond ( $\mathrm{fs}_{\mathrm{s}}$ laser followed by the pulse compressor, AOM, and the confocal microscope setup. The AOM is used to modulate the power delivered to the sample using a diffraction grating. The laser beam reaches the sample, and the SHG and TPEF signals from the samples pass through a bandpass (BP) and short-pass (SP) filter in transmission and reflection modes, respectively. The signals are collected using a photomultiplier tube (PMT). (a), (b), and (c) are three positions at which the laser beam is characterized using the chirp analyzer. The laser beam from the femtosecond laser, (a) before the AOM, (b) after the AOM, and (c) at the sample stage is guided to the chirp analyzer using mirrors (M1 to M6) and a beamsplitter. The beamsplitter (BS) is used to reduce the laser power by $50 \%$ in (a) and to split the beams orthogonally in (c), and then is used to guide by means of mirrors to the chirp analyzer.

ity of PM-SHIM to quantify collagen in tumor allowed us to directly test the hypothesis that chemotherapy can increase collagen fibers inside tumors to impede further therapeutic efficacy of chemotherapeutic agents.

\section{Materials and Methods}

\subsection{Imaging System}

The light source used is an ultrafast laser operating at $900 \mathrm{~nm}$ with 100-fs pulse duration (Mai Tai Titanium Sapphire Laser, Spectraphysics, Newport Corporation, Irvine, California). The imaging was performed using a confocal microscope (LSM 510 Meta, Carl Zeiss GmbH, Jena, Germany). A source laser passes through the prism-based pulse compressor (Femto Control, APE GmbH, Berlin Germany) followed by the acousto-optic modulator (AOM), and is then focused on the sample with a $20 \times$ objective lens with numerical aperture $(\mathrm{NA})=0.5$. The SHG signal was measured on the other side of the sample, filtered through a 450-nm bandpass (BP) filter (full-width half-maximum $=10 \mathrm{~nm}$ ) before reaching the photomultiplier tube (PMT) (R6357, Hamamatsu Photonics, Hamamatsu City, Japan). The schematic of the microscope setup is shown in Fig. 1(a).

A chirp analyzer (GRating-Eliminated No-nonsense Observation of Ultrafast Incident Laser Light E-fields, Swamp Optics, Atlanta, Georgia) was used for measuring the dispersion profile of the laser resulting from pulse compression. ${ }^{37}$ The beam profile was measured at three locations: 1 . before the AOM, 2. after the AOM, and 3. at the sample stage [see Fig. 1(a)]. The optical setups for all the measurements are shown in Fig. 1(b). For all positions, pulse duration was optimized 
by systematically adjusting the prism positions inside the pulse compressor.

\subsection{Xenograft Model, Tissue Isolation, and Collagen Hydrogel Preparation}

\subsubsection{Cell culture}

Acute myeloid leukemia MV4-11 cells were cultured with RPMI1640 (Invitrogen, Carlsbad, California) supplemented with the addition of $10 \%$ fetal bovine serum (FBS, JRH Bioscience Incorporated, Lenexa, Kansas) at a density of 2 to $10 \times 10^{5}$ cells $\mathrm{ml}^{-1}$ in a humid incubator with $5 \% \mathrm{CO}_{2}$ at $37^{\circ} \mathrm{C}$.

\subsubsection{Animals}

Female severe combined immunodeficiency (SCID) mice (17 to $20 \mathrm{~g}, 4$ to 6 weeks old) were purchased from Animal Resources Centre (Canning Vale, Australia). Exponentially growing MV4-11 cells $\left(5 \times 10^{6}\right)$ were subcutaneously injected into loose skin between the shoulder blades and left front leg of 14 recipient mice, inducing one tumor per mice. All treatment was started 25 days after cell injection, when the mice had palpable tumors of 300 to $400 \mathrm{~mm}^{3}$ average size. ABT- 869 was administrated at $15-\mathrm{mg}-\mathrm{kg}^{-1}$ day $^{-1}$ by oral gavage daily. ABT-869 was provided by Abbott Laboratories (Chicago, Illinois) and prepared as published previously. ${ }^{38}$ The length $\mathrm{L}$ and width $\mathrm{W}$ of the tumor was measured using a vernier caliper, and the tumor volume was calculated as per the formula $\left(\mathrm{L} \times \mathrm{W}^{2}\right) / 2$. The tumor volume was measured prior to (on days 25 and 28) and during the course of treatment (on days 30, 32, and 35). The protocol was reviewed and approved by the Institutional Animal Care and Use Committee (IACUC) in compliance with the guidelines on the care and use of animals for scientific purposes (protocol number 050118). After completion of treatment, animals (seven treated, seven control) were anesthetized using a 90-mg ketamine and 9-mg xylazine mixture. The skin flap was opened, and the chest cavity was exposed to perform a cardiac perfusion of saline to flush out blood and then $4 \%$ paraformaldehyde (PFA) to fix the tissues. One tumor per mice, the liver, and some muscle tissue were isolated and frozen immediately in liquid nitrogen. Tissues were sectioned at $40 \mu \mathrm{m}$ for imaging and $5 \mu \mathrm{m}$ for histology. Collagen hydrogel was prepared by mixing $0.49-\mathrm{ml}$ rat tail type- 1 collagen (BD Biosciences, San Jose, California), 100- $\mu$ l phosphate buffered saline (PBS), and $0.41-\mathrm{ml} 0.025-\mathrm{M}$ sodium hydroxide to obtain a 4- $\mathrm{mg} \mathrm{ml}^{-1}$, pH 7.4 neutralized collagen solution. $100 \mu \mathrm{l}$ of the solution was pipetted onto a 0.17 -mm-thick coverslip and dried at room temperature for $48 \mathrm{~h}$ before imaging.

\subsection{Histology}

The sliced tissue samples were stained with a Masson Trichrome (MT) stain kit (ChromaView advanced testing, 87019, Richard-Allan Scientific, Thermo Fisher Scientific, Wathan, Massachusetts) and imaged (IX51, Olympus).

\subsection{Image Acquisition and Signal-to-Noise Ratio Analysis}

Conventional SHG images were taken without pulse compression, in which the laser bypasses the pulse compressor prisms and is routed directly to the microscope. PM-SHIM images were taken when the pulse duration was minimized with optimized pulse compression. All samples, including muscle, liver, and collagen hydrogels, were imaged with both conventional SHIM and PM-SHIM. The average laser power outside the laser was $1.42 \mathrm{~W}$ and attenuated to $50 \mathrm{~mW}$ at the sample stage. The PMT voltage was set at $900 \mathrm{~V}$ for all recordings. In all cases, we recorded a background image using plain glasses as dark background levels for signal processing. We used the $20 \times$ objective to obtain $460 \times 460-\mu \mathrm{m}, 512$ $\times 512$ pixel images. Nine such images were stitched to obtain the tile scan image of $1382 \times 1382-\mu \mathrm{m}, 1536 \times 1536$ pixel images. The tile scan images were used for analysis purposes. SNR was defined as the average pixel intensity value of SHG signal to the background intensity acquired earlier from the plain glass. SNR of conventional SHIM and PM-SHIM was compared directly by dividing one SNR with the other. The SNR calculation was repeated 50 times, and an average SNR and standard deviation was calculated.

\subsection{Image Acquisition and Quantification of Collagen Remodeling in Tumor Samples}

PM-SHIM and conventional SHIM images of the tumor sample were acquired using a $20 \times$ objective. Nine images of $512 \times 512$ pixels, $460 \times 460 \mu \mathrm{m}$ were taken per tumor sample in the tumor interior $200 \mu \mathrm{m}$ from the tumor boundary to avoid the collagen concentration spikes in the tumor boundary. Images were acquired at a depth of $20 \mu \mathrm{m}$ in the $40-\mu \mathrm{m}$ tissue section. The tissue section imaged was sliced about $1 \mathrm{~mm}$ deep into the tumor. An image segmentation algorithm based on a mixture Gaussian model was performed to remove background and noise. It is assumed that the intensity of pixels in the image can be modeled as the mixture of two Gaussian distributions, one representing the collagen area with strong SHG signals, and the other representing the background. Using the expectation-maximization (EM) algorithm, ${ }^{39}$ the parameters of the Gaussian distributions that model the peak intensity of pixels in the image was found. A binary image was generated by applying a value of 1 to all pixels having intensity that belongs to the Gaussian distribution representing the collagen area, and a value of 0 to the rest of the pixels.

We quantified four parameters, namely collagen area percentage, fiber number, fiber length, and fiber width. The percentage of collagen area was determined as the number of pixels that are segmented as collagen divided by the total number of pixels in the same image. After collagen segmentation, the distance transform is first performed on the binary image, which calculates the distance from a fiber pixel to a background pixel. After thresholding of the distance function, global maximum points are identified as the cross-link points. Starting from these cross-link points, fibers are traced through several local maxima points until the end of the fiber or another cross-link point is reached. The skeleton of each fiber in the image is extracted by connecting cross-link points and local maxima points. Then, the number of fibers, average fiber 
(a)

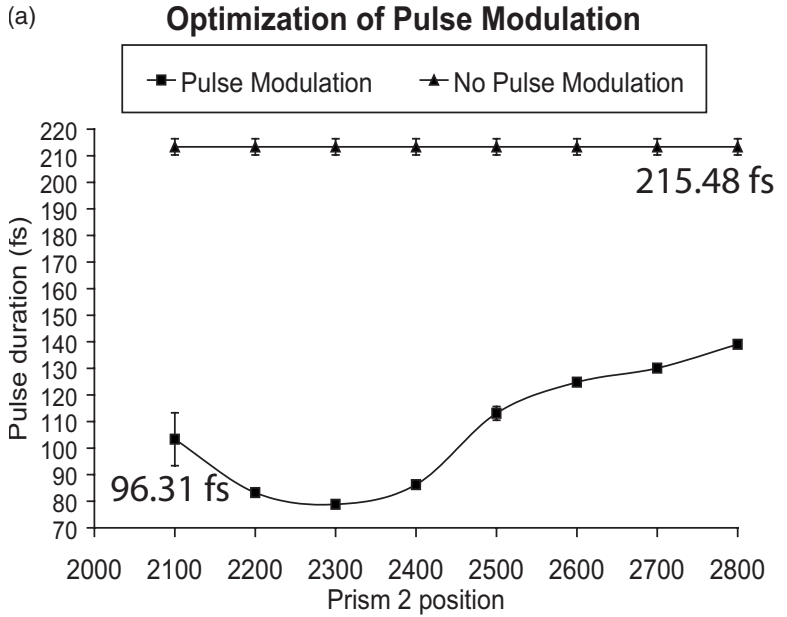

(b)

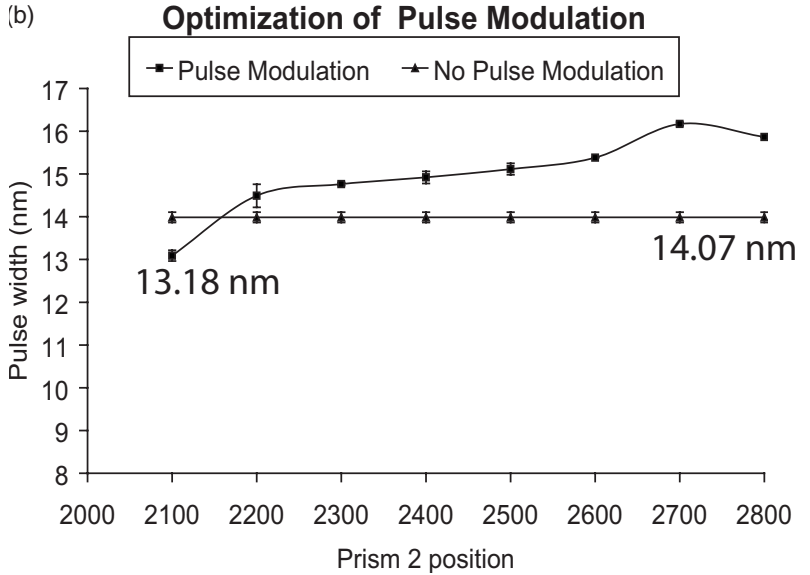

(c)
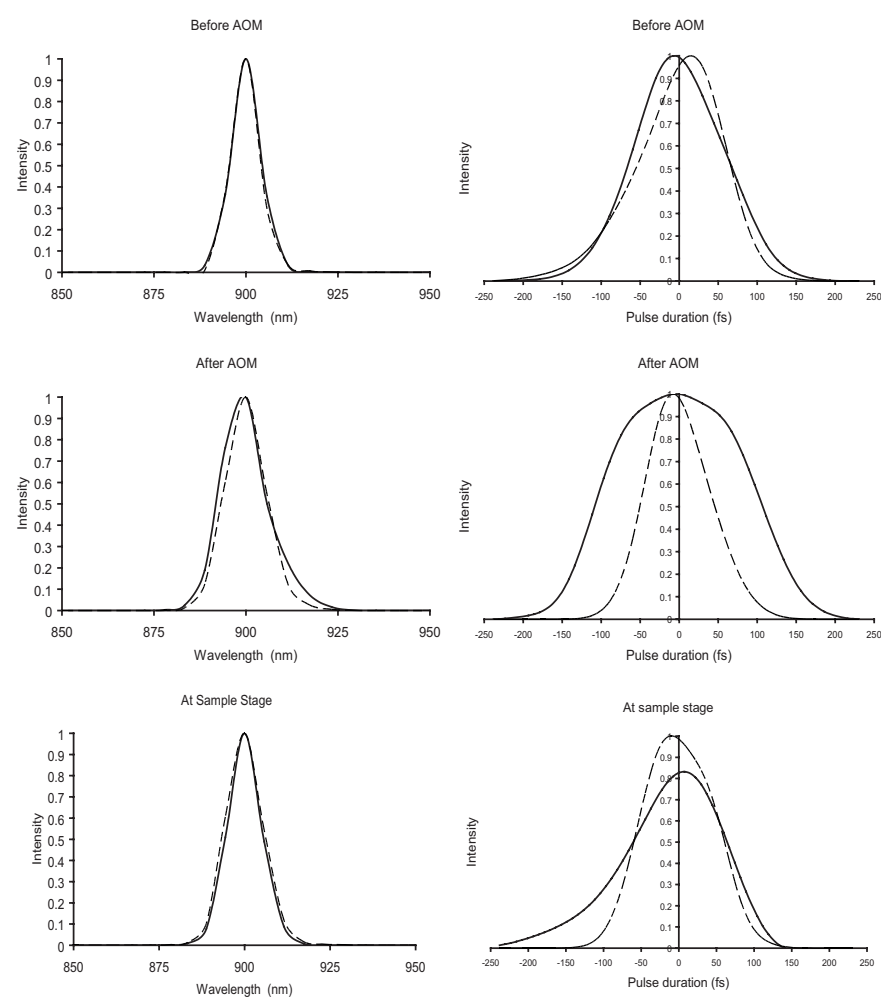

Fig. 2 Chirp analyses of the laser beam of the PM-SHIM for optimization of prism positions in the pulse compressor. (a) Pulse duration in femtosecond ( $\mathrm{fs}$ ) and (b) pulse width in nanometers for different prism positions with pulse modulation (boxes) and without pulse modulation (triangles) are shown. The maximum pulse duration after dispersion is $215.48 \mathrm{fs}$, and the minimum pulse duration after modulation is $96.31 \mathrm{fs}$. The optimized prism positions are 1100 for prism 1 and 2100 for prism 2. (c) Spatial profile-pulse width in nanometers (left column), and temporal profile-pulse duration in fs (right column) of the laser beam before AOM, after AOM, and at the sample stage are shown for PM-SHIM (dotted line) and conventional SHIM (solid line). The dispersion affects the pulse duration rather than the pulse width.

length, and average fiber width are quantified. ${ }^{40}$ All image processing and algorithm execution were carried out using MATLAB (The Math Works, Incorporated, Natick, Massachusetts). The image processing algorithm code is available for readers on request.

\section{Results and Discussion}

We systematically optimized the PM-SHIM by using a chirp analyzer to characterize both the spatial and temporal profiles to determine the optimal pulse compression. With the optimized PM-SHIM, we observed a significant SNR improvement, as high as 3.2 times, in all biological samples. In addition, we could visualize and differentiate the collagen fibers in the drug-treated and control tumor samples. We have directly observed that collagen quantity inside tumors increased significantly in the drug-treated group compared to the control group, and found a distinct difference in morphological features from both groups. These findings are being reported for the first time because of the superior excitation and detection sensitivity in PM-SHIM over the conventional SHIM, and they correlate well with the deterioration of the chemotherapeutic effects of anticancer compounds in animal models over time.

\subsection{Pulse Compressor Optimization}

In Fig. 2, the optimization of prism positions in the pulse compressor to obtain the best pulse modulation is shown. Figure 2(a) shows the pulse duration plots and Fig. 2(b) shows the pulse width plots at various prism positions. The optimized prism 1 position was found to be 1100 and the optimized prism 2 position was 2100 . The spectral and temporal profiles of the beam measured at three different locations are shown in Fig. 2(c), in which the beam profile in the PMSHIM and conventional SHIM are shown in dotted and solid lines, respectively. The pulse width reduced from 14 to $13.18 \mathrm{~nm}$ in the PM-SHIM, and the pulse duration improved from $215 \mathrm{fs}$ (conventional SHIM) to $96 \mathrm{fs}$ (PMSHIM). We improved the peak power delivered to the sample from 2.3 to $6.5 \mathrm{KW}$ after pulse modulation. We can also see that dispersion introduced by the optical components affects 

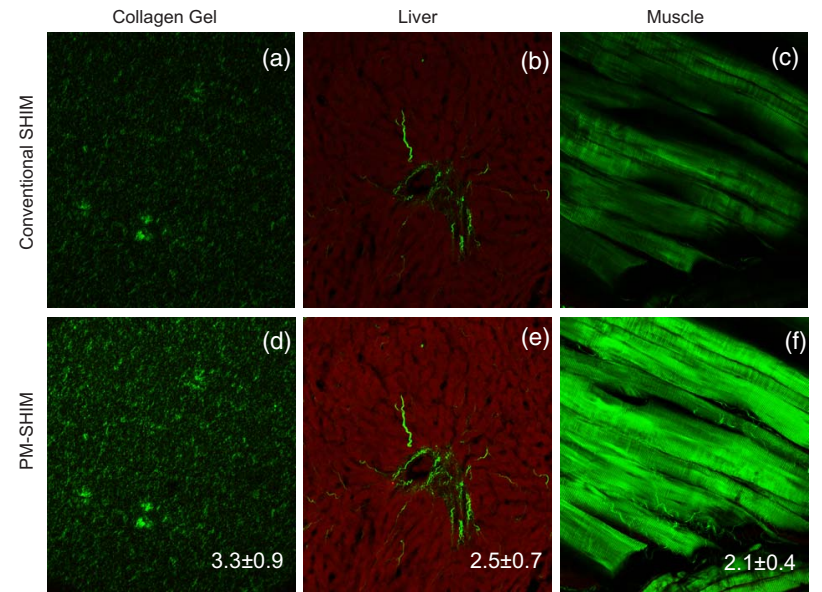

Fig. 3 Collagen gels, liver, and muscle samples exhibit improved SNR with PM-SHIM. Samples demonstrating the improvement of SHG and TPEF signals in collagen gels [(a) and (d)], liver tissue slice [(b) and $(\mathrm{e})]$, and mouse thigh muscle [(c) and (f)]. The degree of SNR improvement is indicated on the improved images in the right bottom corner. The visualization of small collagen fibers in the liver parenchyma (e) and the small collagen fibrils in the muscle sample (f) is made possible with pulse modulation. Scale bar: $50 \mu \mathrm{m}$.

mostly the pulse duration instead of the pulse width. The pulse duration measurement at the sample stage was taken from the reflection of the signal from the sample stage. In effect, the light is traveling through the microscope components twice, and the pulse duration measured at the end point was $115 \mathrm{fs}$. Thus, the pulse duration of the beam reaching the sample is estimated to be less than 115 fs and more than 96 fs (pulse duration measured before entering the microscope).

\subsection{Signal-to-Noise Ratio Improvement in the Pulse-Modulated Second Harmonic Imaging Microscope}

SHG images from collagen gels, liver, and muscle sections in the conventional SHIM and PM-SHIM are shown in Fig. 3. The SHG image obtained from the collagen fibers in the gel construct using the conventional SHIM [Fig. 3(a)] is not clear, while those obtained using PM-SHIM are brighter and sharper [Fig. 3(d)]. Figures 3(b) and 3(e) show the portal triads as well as the liver parenchyma in liver lobules imaged with conventional SHIM and PM-SHIM. The SHG signal generated by collagen is shown in the green channel, and the twophoton excited fluorescence (TPEF) in the hepatocytes is shown in the red channel. The smaller collagen fibers in the liver parenchyma are clearly visualized with PM-SHIM but not with SHIM. Similarly, with mouse thigh muscle, the individual muscle fibers and the collagen fibrils surrounding the muscle fibers cannot be visualized with conventional SHIM [Fig. 3(c)], but only with PM-SHIM [Fig. 3(f)].The ratio of SNR from PM-SHIM and SHIM is shown in the lower right corners of the images. On average, there is a $3.3 \pm 0.9$-fold improvement for collagen gels, $2.5 \pm 0.7$-fold increase for liver tissue, and 2.1 \pm 0.4 -fold SNR improvement in muscle samples.

We have demonstrated a marked improvement for collagen visualization with more than two-fold improvement in SNR. As SHG is a stain-free imaging system, the SHG signal inten-

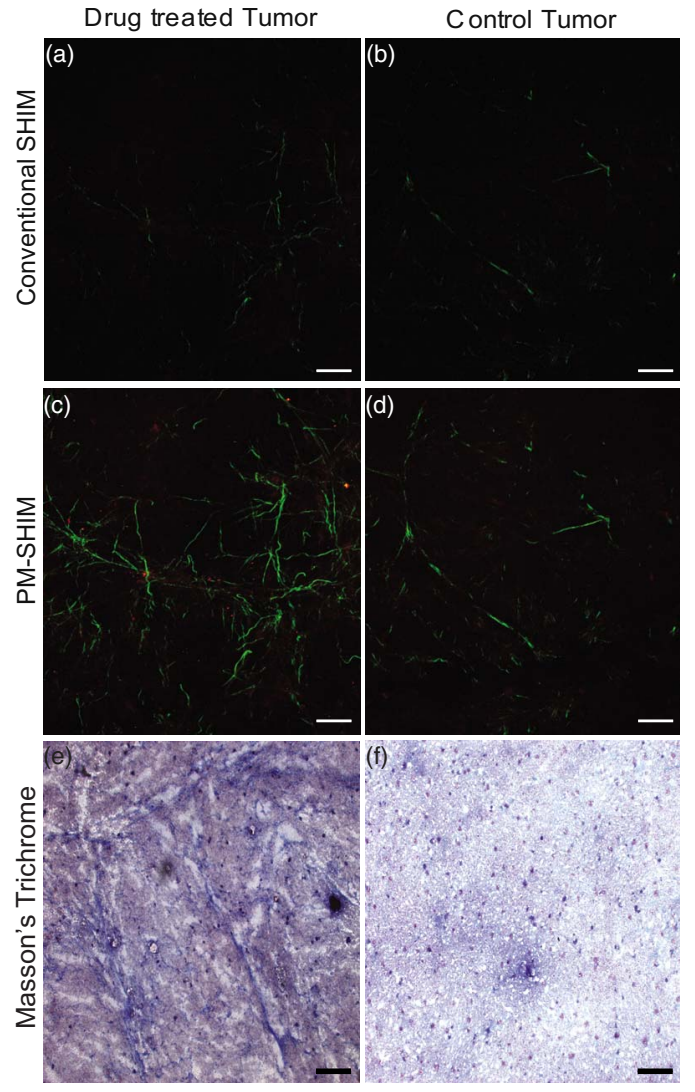

Fig. 4 Collagen fibers in chemotherapy drug-treated samples can be clearly visualized using the PM-SHIM. Representative images of tumor samples before and after chemotherapy are shown. (a) and (b) show images taken with conventional SHIM, (c) and (d) show images taken with PM-SHIM, and (e) and (f) show the Masson's trichrome stained slides of the drug-treated and control samples, respectively. The laser power used to excite the samples with conventional SHIM was $10 \%$ higher than with PM-SHIM. The smaller fibers are visualized by the PM-SHIM, which are not excited with conventional SHIM. Scale bar: $50 \mu \mathrm{m}$.

sity observed correlates directly to the collagen amount present in the sample rather than the quantity of dye present in the sample. It also helps in rapid sample preparation, making it an easy technique for imaging biopsy samples, where the tissue can be imaged using PM-SHIM and then used for other routine histology techniques.

\subsection{Collagen Modulation on Drug Administration Visualized with Pulse-Modulated Second Harmonic Imaging Microscope}

The in-vivo activity of ABT-869 on MV4-11 xenograft tumors was evaluated previously. ${ }^{1}$ The tumors were reduced to unpalpable size but the tumor cells were not completely eliminated by the drug treatment. In the PM-SHIM, the collagen fiber distribution in the drug-treated and control group was clearly visualized [Figs. 4(a) and 4(b)], while in the conventional SHIM very few collagen fibers can be visualized in the tumor stroma, even with maximized laser power and detector sensitivity [Figs. 4(c) and 4(d)]. The Masson's Trichrome stain reveals some differences in collagen distribution between 
(a)

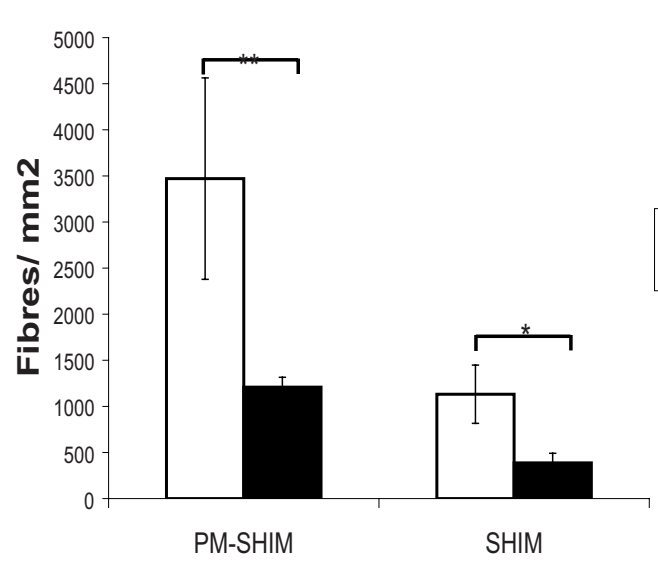

(b)
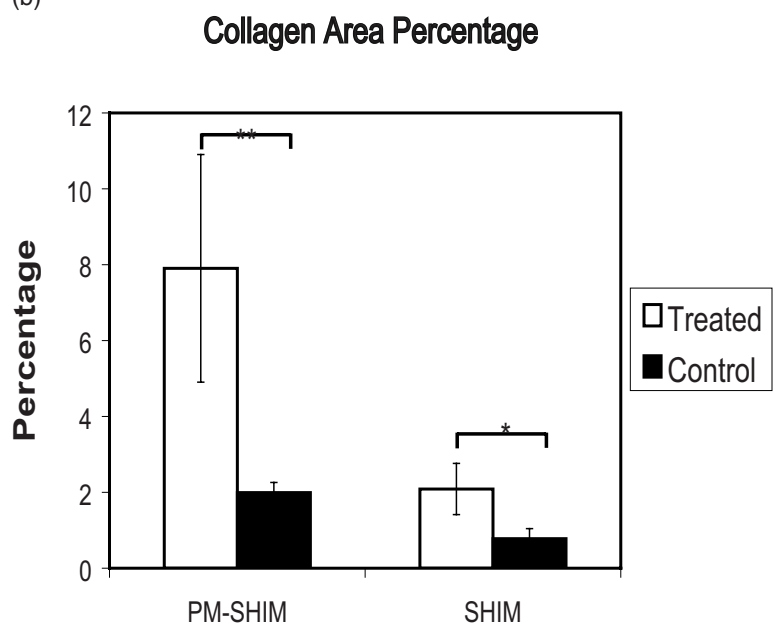

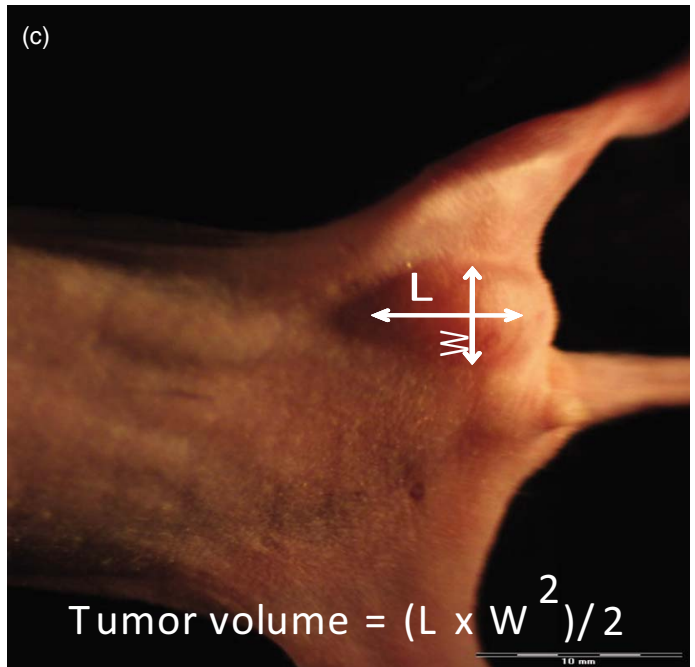

(d)

Tumor volume measur em ents during

ABT-869 tr eatment

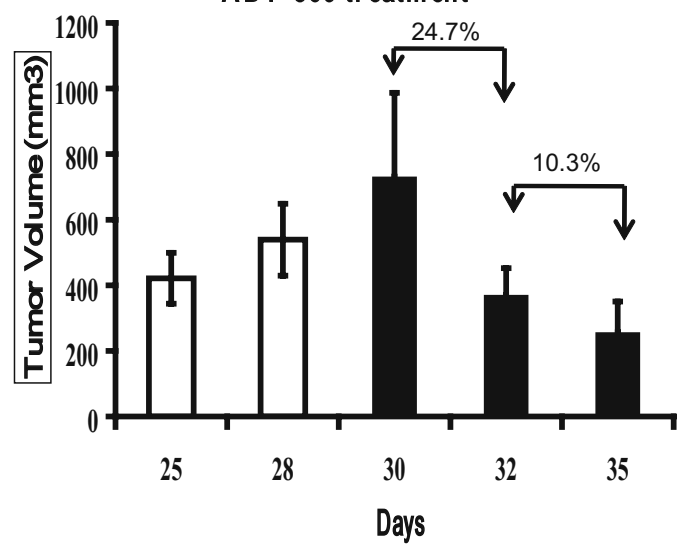

Fig. 5 Quantification of collagen properties in drug-treated samples shows improved fiber number and collagen area percentage with PM-SHIM. The collagen fiber number and area percentage are shown in (a) and (b). The two parameters are plotted for the drug-treated (white bars) and control (black bars) samples with PM-SHIM and with conventional SHIM. Statistical significance was tested with a Student's t-test, $p<0.0002 * *$ for collagen fiber number and $p<0.0004 * *$ for collagen area percentage with PM-SHIM, and $p<0.079 *$ and $p<0.094 *$, respectively, for conventional SHIM. The difference in the collagen parameters between the drug-treated and control samples can be clearly visualized using the PM-SHIM. The formula used to measure the tumor volume is shown in (c). The tumor volume changes before and after ABT-869 treatment are shown in (d). The percentage volume change per day between the data points are marked in the graph.

treated and control samples [Figs. 4(e) and 4(f)] in semiquantitative manners. ${ }^{41,42}$

Collagen fiber contents in the tumors were quantitatively analyzed comparing nine images $(460 \times 460 \mu \mathrm{m}$ each $)$ from seven treated and seven control tumors. The collagen fiber number and collagen area percentage of the treated and control tumors are shown in Fig. 5. The drug-treated group is shown by the white bar and the control group by the black bar for both PM-SHIM and conventional SHIM systems. We have found that by using PM-SHIM, the number of collagen fibers is much higher in the drug-treated group [Fig. 5(a), $3470.8 \pm 1092$ fibers $/ \mathrm{mm}^{2}$ ] than the control group [Fig. 5(a), $1131.7 \pm 315$ fibers $\left./ \mathrm{mm}^{2}\right]$ with $p<0.0002$. In the conventional SHIM, the fiber numbers were 1208.6 \pm 107.3 fibers $/ \mathrm{mm}^{2}$ for the treated group and $386.9 \pm 104$ fibers $/ \mathrm{mm}^{2}$ for the control group with $p$ $<0.079$. As shown in Fig. 5(b), by using PM-SHIM the col- lagen area percentage of the drug-treated group was $7.9 \pm 3 \%$, while that of the control group was $2.0 \pm 0.2 \%$. In the conventional SHIM, the percentages were $2.1 \pm 0.7$ and $0.8 \pm 0.3 \%$ for the treated and control groups, respectively. On comparing the treated and control samples using a Student's t-test, the percentage calculated from the PM-SHIM images showed a statistical significance of $p<0.0004$, and for the conventional SHIM it was $p<0.094$.

On quantifying the collagen fiber lengths and widths, we found that we were able to detect longer and wider fibers in the treated group using PM-SHIM. The longest fiber we detected in the treated group using PM-SHIM was $155.2 \mu \mathrm{m}$, while that of the fibers visualized using conventional SHIM was $48.8 \mu \mathrm{m}$. The longest fiber for the control group visualized using PM-SHIM was $55.7 \mu \mathrm{m}$, and that using conventional SHIM was $32.9 \mu \mathrm{m}$. Similarly, the widest fiber we de- 
tected in the treated group using PM-SHIM was $77 \mu \mathrm{m}$, while that of the fibers visualized using conventional SHIM was only $17 \mu \mathrm{m}$. The longest fiber for the control group visualized using PM-SHIM was $12.6 \mu \mathrm{m}$, and that using conventional SHIM was $11.7 \mu \mathrm{m}$. This shows that there were several disconnects in the fibers visualized using conventional SHIM, hence segmenting the same fiber into smaller, thinner fibers. Even though the fibers were segmented, the overall number of fibers detected was not elevated in the conventional SHIM, as many of the fiber signals were too weak to be detected.

The frequency distributions of the length and width of the fibers for the treated group and control group imaged with PM-SHIM and conventional SHIM (solid squares and triangles) are shown in graphs 1 and 2 on the left side of Fig. 6, respectively. Regions of the plot are enlarged to show clearly the length and width distribution of the fibers in Figs. 6(a)-6(d). It is evident from the enlarged graphs that only the length and width distribution of PM-SHIM is distinguishable between the treated and control groups.

In our tumor volume measurements [method to calculate tumor volume is shown in Fig. 5(c)], we find that the percent tumor volume reduction is $24.7 \%$ per day in the first two days of treatment, while it decreases to $10.3 \%$ per day in the next three days of treatment, as shown in Fig. 5(d). This drop in percent of tumor volume reduction per day could indicate reduction of efficacy of chemotherapy due to hindrance caused by the wound-healing response. This correlates with our data that collagen increases after treatment.

The fibers observed in the control group include a few thick and long fibers, representing more mature fibers, and some scattered small and thin fibers that appear as speckles, representing less mature fibers or degrading fibers. In the drug-treated group, we observe branched and shorter fibers connected to the long mature fibers, and the fibers appeared to be brighter in general. Not many speckled collagen structures were observed in the drug-treated group. The reduced speckle content in the drug-treated sample could indicate lower degradation of the collagen fiber. The brighter short fibers could indicate more collagen production, and the long thick fibers represent fiber maturation in the drug-treated group. ABT-869 is a multitargeted receptor tyrosine kinase inhibitor targeting mainly the vascular endothelial growth factor receptors (VEGFR) and platelet derived growth factor receptors (PDGFR). PDGFRs have been shown to activate collagen production in sclerosis models. ${ }^{43}$ Thus, ABT-869 blocking PDGFRs should theoretically down-regulate collagen production, which cannot explain the observed up-regulation of collagen production in the tumors. It is more likely that the chemotherapy triggers a wound-healing response, resulting in the production of new collagen fibers and reduced degradation of the existing fibers.

One of the hallmarks of tumor progression is reduced expression of extracellular matrix, especially collagen type $1 .^{44}$ The collagen in the tumor interior is reduced, while at the tumor boundary the area of collagen (collagen cap) is increased. The increase in collagen in the cap has been attributed to the pushing of the pre-existing collagen bundles by the cancer cells onto the surrounding normal tissue. ${ }^{45}$ The collagen cap is further bolstered by collagen production by acti- vated fibroblasts. The collagen cap acts as a barrier to drugs, ${ }^{24}$ but when needed the barrier is broken down by metastasizing cancer cells. ${ }^{46}$ In the tumor interior, the collagen fibers are thin and sparse, as they are all newly synthesized by activated fibroblasts and cancer cells but are not part of existing collagen bundles.

During chemotherapy, when the drug diffuses beyond the collagen cap and reaches the tumor interior, several genes in the fibroblasts and cancer cells can be activated to release factors that might render the cancer cells resistant to chemotherapy. There are studies pointing to this effect of chemotherapy on activated stromal cells releasing factors, such as hyaluronic acid, ${ }^{47}$ integrins, and fibronectins, ${ }^{48,49}$ that are often associated with local wound-healing processes. A study by Farmer et al. ${ }^{50}$ showed that a distinct increase in the expression of stromal signature genes predicts resistance to chemotherapy in biopsy samples. However, none of these hypotheses have been directly tested by investigating the tumor responses to chemotherapy. In our study, we have quantified an increase in collagen fibers in the tumor interior after chemotherapy, which might be due to the activated stromal cells involved in local wound healing.

Furthermore, this increased collagen in the tumor interior can activate TGF- $\beta$, a master cytokine which in turn affects the fibroblast growth factor (FGF), platelet derived growth factor (PDGF), ${ }^{51}$ insulin-like growth factor (IGF), and Interleukin- $6{ }^{52}$ These factors exert compounding effects on the proliferation, activation, and transformation of stromal and cancer cells. The collagen increase in the tumor interior can also increase the mechanical stiffness of the tissue microenvironment, which favors cancer cell proliferation. ${ }^{53} \mathrm{Fi}$ nally, the additional collagen fibers can bind to proangiogenic factors, preventing new vessel formation, ${ }^{54}$ and thus further limiting the access of chemotherapeutic agents to the remaining cancer cells. Therefore, the observed increase in collagen in the tumor interior could impede sustained efficacy of chemotherapy through more complex mechanisms than previously postulated, based purely on the ECM modulation observed in the tumor boundary. ${ }^{24}$ PM-SHIM provides us with a quantitative tool to further investigate these mechanisms. PMSHIM can also enable us to design new regimens of drug treatment, including collagen-modulating components introduced at the appropriate time to reduce collagen hindrance and promote drug penetration.

We have demonstrated that the collagen content in the tumor interior is distinctly different after chemotherapy. The mature fibers in the tumor interior can be visualized with the conventional SHIM, albeit with reduced signal intensity, but the small immature fibers that contribute to a considerable amount of collagen area are only visible in the PM-SHIM images. Thus using the PM-SHIM, we obtained accurate quantification of collagen area percentage, fiber number, and collagen fiber length and width, allowing us to draw statistically significant conclusions about the drug effects on tumors.

\section{Conclusion}

Pulse modulation can improve the SNR and pulse characteristics in second harmonic imaging, which helps in detecting subtle changes in collagen signals inside the tumor interior that are difficult to detect by conventional SHIM. We quantify 
Raja et al.: Pulse-modulated second harmonic imaging microscope quantitatively demonstrates..
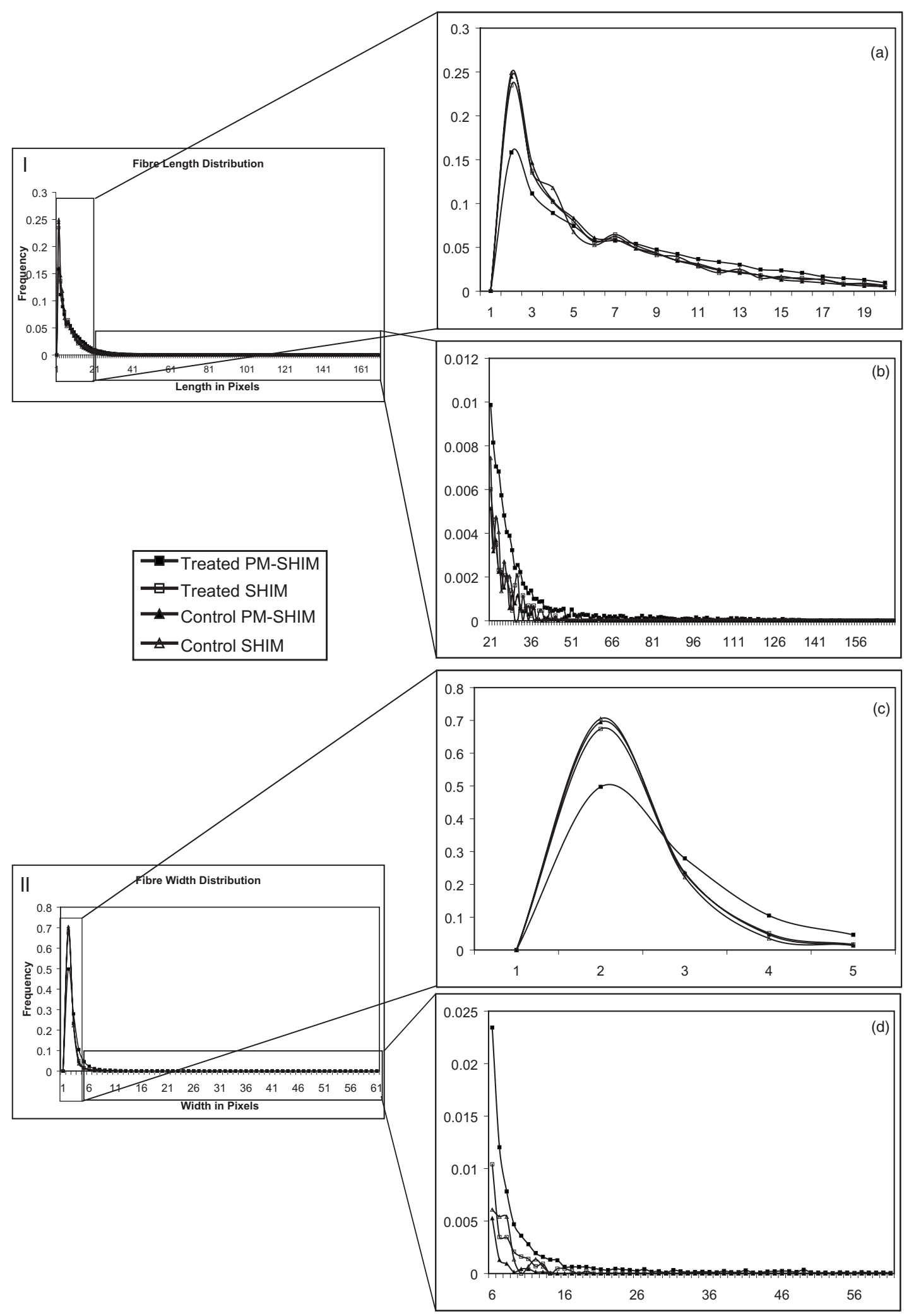

Fig. 6 Quantification of collagen fiber length and width shows distinction between the treated and control samples with PM-SHIM (solid squares). Graphs 1 and 2 depict the length and width frequency distribution of the treated and control group visualized using the PM-SHIM (solid squares and triangles) and the conventional SHIM (empty squares and triangles). The enlarged view of collagen fiber length distribution is shown in (a) and (b) and the collagen fiber width in (c) and (d). 
with PM-SHIM the spatial distribution of collagen inside a xenograft model of human acute myeloid leukemia tumor specimens treated with ABT-869, and detect a significant increase in collagen synthesis inside tumor after chemotherapy that can partially account for resistance to chemotherapy. PMSHIM can also help in the design of improved chemotherapeutic regimens or combination therapies involving collagenmodulating components to eliminate the last trace of cancer cells in tumors.

\section{Acknowledgments}

This work is supported in part by the Institute of Bioengineering and Nanotechnology, BMRC, A ${ }^{*}$ STAR of Singapore, and grants from BMRC (R185-001-045-305), Ministry of Education (R-185-000-135-112), Janssen-Cilag (R-185-000-182592), Singapore-MIT Alliance Computational and Systems Biology Flagship Project funding (C-382-603-004-001), SMART BioSyM and Mechanobiology Institute, Singapore to Yu. Raja and Zhou are NUS Research Scholars, and Xu is a SMA Research Scholar.

\section{References}

1. J. Zhou et al., "Synergistic antileukemic effects between ABT-869 and chemotherapy involve downregulation of cell cycle-regulated genes and c-Mos-mediated MAPK pathway," Leukemia 22(1), 138146 (2008).

2. H. F. Dvorak, J. Flier, and H. Frank, "Tumors-wounds that do not heal-similarities between tumor stroma generation and wound healing," N. Engl. J. Med. 315(26), 1650-1659 (1986).

3. J. Hay, S. Shahzeidi, and G. Laurent, "Mechanisms of Bleomycininduced lung damage," Arch. Toxicol. 65(2), 81-94 (1991).

4. H. Tsuchiya, Y. Ueda, K. Tomita, I. Nakanishi, and A. Roessner, "Chemotherapeutci effect on osteosarcoma on basis of collagen analysis - a proposal of the induction of osteosarcoma differentiation," J. Cancer Res. Clin. Oncol. 119(12), 702-706 (1993).

5. R. K. Jain, D. G. Duda, C. G. Willett, D. V. Sahani, A. X. Zhu, J. S Loeffler, T. T. Batchelor, and A. G. Sorensen, "Biomarkers of response and resistance to antiangiogenic therapy," Nature Rev. Clin. Oncol. 6(6), 327-338 (2009).

6. P. J. Campagnola, A. C. Millard, M. Terasaki, P. E. Hoppe, C. J Malone, and W. A. Mohler, "Three-dimensional high-resolution second-harmonic generation imaging of endogenous structural proteins in biological tissues," Biophys. J. 82(1), 493-508 (2002).

7. R. M. Williams, D. W. Piston, and W. W. Webb, "2-photon molecular excitation provides intrinsic 3-dimensional resolution for laser-based microscopy and microphotochemistry," FASEB J. 8(11), 804-813 (1994).

8. S. J. Lin, J. S. Kuo, C. J. Wu, R. J. Lin, W. C. Chen, J. S. Liao, Y. H Hsu, C. J. Tsai, T. F. Chen, and D. C. Yu, "Discrimination of basal cell carcinoma from normal dermal stroma by quantitative multiphoton imaging," Opt. Lett. 31(18), 2756-2758 (2006).

9. P. Wilder-Smith, W. G. Jung, J. Zhang, Z. Chen, K. Osann, and B Tromberg, "Noninvasive imaging of oral premalignancy and malignancy," J. Biomed. Opt. 10(5), 051601 (2005).

10. P. J. Campagnola, M.-d. WeiA. Lewisand L. M. Loew“Highresolution nonlinear optical imaging of live cells by second harmonic generation," Biophys. J. 77(6), 3341-3349 (1999).

11. J. G. Lyubovitsky, T. B. Krasieva, X. Xu, B. Andersen, and B. J. Tromberg, "In situ multiphoton optical tomography of hair follicles in mice," J. Biomed. Opt. 12(4), 044003 (2007).

12. D. W. Piston, "Imaging living cells and tissues by two-photon excitation microscopy," Trends Cell Biol. 9(2), 66-69 (1999).

13. C. Soeller and M. B. Cannell, "Construction of a two-photon microscope and optimisation of illumination pulse duration," Pflugers Arch. Eur. J. Physiol. 432(3), 555-561 (1996).

14. T. Ragan, J. D. Sylvan, K. H. Kim, H. Huang, K. Bahlmann, R. T. Lee, and P. T. C. So, "High-resolution whole organ imaging using two-photon tissue cytometry," J. Biomed. Opt. 12(1), 014015 (2007).
15. Q. F. Wu and A. T. Yeh, "Rabbit cornea microstructure response to changes intraocular pressure visualized by using nonlinear optical microscopy," Cornea 27(2), 202-208 (2008).

16. M. Strupler, A.-M. Pena, M. Hernest, P.-L. Tharaux, J.-L. Martin, E. Beaurepaire, and M.-C. Schanne-Klein, "Second harmonic imaging and scoring of collagen in fibrotic tissues," Opt. Express 15(7), 40544065 (2007).

17. N. D. Kirkpatrick, S. Andreou, J. B. Hoying, and U. Utzinger, "Live imaging of collagen remodeling during angiogenesis," Am. J. Physiol. Heart Circ. Physiol. 292(6), H3198-H3206 (2007).

18. M.-H. Chen, W.-L. Chen, Y. Sun, P. T. Fwu, and C.-Y. Dong, "Multiphoton autofluorescence and second-harmonic generation imaging of the tooth," J. Biomed. Opt. 12(6), 064018 (2007).

19. P. P. Provenzano, C. T. Rueden, S. M. Trier, L. Yan, S. M. Ponik, D. R. Inman, P. J. Keely, and K. W. Eliceiri, "Nonlinear optical imaging and spectral-lifetime computational analysis of endogenous and exogenous fluorophores in breast cancer," J. Biomed. Opt. 13(3), 031220 (2008)

20. P. P. Provenzano, K. W. Elicein, L. Yan, A. Ada-Nguema, M. W. Conklin, D. R. Inman, and P. J. Keely, "Nonlinear optical imaging of cellular processes in breast cancer," Microsc. Microanal. 14(6), 532_ 548 (2008)

21. S. M. Zhuo, J. X. Chen, T. S. Luo, X. S. Jiang, S. S. Xie, and R. Chen, "Two-layered multiphoton microscopic imaging of cervical tissue," Lasers Med. Sci. 24(3), 359-363 (2009).

22. P. P. Provenzano, K. W. Eliceiri, J. M. Campbell, D. R. Inman, J. G. White, and P. J. Keely, "Collagen reorganization at the tumor-stromal interface facilitates local invasion," Bmc Med. 4 (2006).

23. N. D. Kirkpatrick, M. A. Brewer, and U. Utzinger, "Endogenous optical biomarkers of ovarian cancer evaluated with multiphoton microscopy," Cancer Epidemiol. Biomarkers Prev. 16(10), 2048-2057 (2007).

24. E. Brown, T. McKee, E. diTomaso, A. Pluen, B. Seed, Y. Boucher, and R. K. Jain, "Dynamic imaging of collagen and its modulation in tumors in vivo using second-harmonic generation," Nat. Med. 9(6), 796-800 (2003).

25. O. Chernyavskiy, L. Vannucci, P. Bianchini, F. Difato, M. Saieh, and L. Kubínová, "Imaging of mouse experimental melanoma in vivo and ex vivo by combination of confocal and nonlinear microscopy," $\mathrm{Mi}$ crosc. Res. Tech. 72(6), 411-423 (2009).

26. X. Han, R. M. Burke, M. L. Zettel, P. Tang, and E. R. Brown, "Second harmonic properties of tumor collagen: determining the structural relationship between reactive stroma and healthy stroma," Opt. Express 16(3), 1846-1859 (2008).

27. P. P. Provenzano, K. W. Eliceiri, and P. J. Keely, "Multiphoton microscopy and fluorescence lifetime imaging microscopy (FLIM) to monitor metastasis and the tumor microenvironment," Clin. Exp. Metastasis 26(4), 357-370 (2009)

28. C.-C. Wang, F.-C. Li, R.-J. Wu, V. A. Hovhannisyan, W.-C. Lin, S.-J Lin, P. T. C. So, and C.-Y. Dong, "Differentiation of normal and cancerous lung tissues by multiphoton imaging," J. Biomed. Opt. 14(4), 044034 (2009).

29. S. M. Zhuo, J. Chen, S. Xie, Z. Hong, and X. Jiang, "Extracting diagnostic stromal organization features based on intrinsic twophoton excited fluorescence and second-harmonic generation signals," J. Biomed. Opt. 14(2), 020503 (2009).

30. R. M. Williams, A. Flesken-Nikitin, L. H. Ellenson, D. C. Connolly, T. C. Hamilton, A. Y. Nikitin, and W. R. Zipfel, "Strategies for highresolution imaging of epithelial ovarian cancer by laparoscopic nonlinear microscopy," Trans. Oncol. 3(3), 181-194 (2010).

31. T. Hompland, A. Erikson, M. Lindgren, T. Lindmo, and C. de Lande Davis, "Second-harmonic generation in collagen as a potential cancer diagnostic parameter," J. Biomed. Opt. 13(5), 054050 (2008).

32. M. Muller, J. Squier, R. Wolleschensky, U. Simon, and G. J. Brakenhoff, "Dispersion pre-compensation of 15 femtosecond optical pulses for high-numerical-aperture objectives," J. Microscopy-Oxford 191, 141-150 (1998)

33. R. L. Fork, O. E. Martinez, and J. P. Gordon, "Negative dispersion using pair of prisms," Opt. Lett. 9(5), 150-152 (1984).

34. V. Iyer, B. E. Losavio, and P. Saggau, "Compensation of spatial and temporal dispersion for acousto-optic multiphoton laser-scanning microscopy," J. Biomed. Opt. 8(3), 460-471 (2003). 
35. S. Tang, T. B. Krasieva, Z. Chen, G. Tempea, and B. J. Tromberg, "Effect of pulse duration on two-photon excited fluorescence and second harmonic generation in nonlinear optical microscopy," $J$. Biomed. Opt. 11(2), 020501 (2006).

36. L. T. Schelhas and M. Dantus, "Advantages of ultrashort phaseshaped pulses for selective two-photon activation and biomedical imaging," Nanomed. 2(3), 177-181 (2006).

37. S. Akturk, M. Kimmel, P. O'Shea, and R. Trebino, "Extremely simple device for measuring 20-fs pulses," Opt. Lett. 29(9), 10251027 (2004).

38. D. B. Shankar et al., "ABT-869, a multitargeted receptor tyrosine kinase inhibitor: inhibition of FLT3 phosphorylation and signaling in acute myeloid leukemia," Blood 109(8), 3400-3408 (2007).

39. A. P. Dempster, N. M. Laird, and D. B. Rubin, "Maximum likelihood from incomplete data via the EM algorithm," J. Royal Stat. Soc. Series B 39(1), 1-38 (1977)

40. A. M. Stein, D. A. Vader, L. M. Jawerth, D. A. Weitz, and L. M Sander, "An algorithm for extracting the network geometry of $3 \mathrm{~d}$ collagen gels," J. Microsc. 232(3), 961-968 (2008).

41. P. Bedossa et al., "Intraobserver and interobserver variations in liverbiopsy intepretation in patients with chronic hepatitis-C," Hepatol. 20(1), 15-20 (1994).

42. A. Theodossi et al. "Observer variation and discriminatory value of biopsy features in inflammatory bowel-disease," Gut 35(7), 961-968 (1994).

43. S. S. Baroni et al., "Stimulatory autoantibodies to the PDGF receptor in systemic sclerosis," N. Engl. J. Med. 354(25), 2667-2676 (2006).

44. H. Green, G. J. Todaro, and B. Goldberg, "Collagen synthesis in fibroblasts transformed by oncogenic viruses," Nature 209(5026), 916 (1966).

45. J. Smolle, M. Fiebiger, R. Hofmann-Wellenhof, and H. Kerl, "Quantitative morphology of collagen fibers in cutaneous malignant melanoma and melanocytic nevus," Am. J. Dermatopathol. 18(4), 358363 (1996)

46. B. R. Lester and J. B. McCarthy, "Tumor-cell adhesion to the extracellular-matrix and signal transduction mechanisms implicated in tumor-cell motility, invasion and metastasis," Cancer Metastasis Rev. 11(1), 31-44 (1992).

47. S. Misra, S. Ghatak, and B. P. Toole, "Regulation of MDR1 expression and drug resistance by a positive feedback loop involving hyaluronan, phosphoinositide 3-kinase, and ErbB2," J. Biolog. Chem. 280(21), 20310-20315 (2005)

48. V. M. Weaver, S. Lelièvre, J. N. Lakins, M. A. Chrenek, J. C. R. Jones, F. Giancotti, Z. Werb, and M. J. Bissell, "Beta 4 integrindependent formation of polarized three-dimensional architecture confers resistance to apoptosis in normal and malignant mammary epithelium," Cancer Cells 2(3), 205-216 (2002).

49. L. A. Hazlehurst, N. Valkov, L. Wisner, J. A. Storey, D. Boulware, D. M. Sullivan, and W. S. Dalton, "Reduction in drug-induced DNA double-strand breaks associated with beta 1 integrin-mediated adhesion correlates with drug resistance in U937 cells," Blood 98(6), 1897-1903 (2001)

50. P. Farmer et al., "A stroma-related gene signature predicts resistance to neoadjuvant chemotherapy in breast cancer," Nat. Med. 15(2), 220-220 (2009).

51. M. J. Newman, "Transforming growth-factor-Beta and the cell surface in tumor progression," Cancer Metastasis Rev. 12(3-4), 239254 (1993)

52. O. Purps, B. Lahme, A. M. Gressner, N. M. Meindi-Beinker, and S. Dooley, "Loss of TGF-beta dependent growth control during HSC transdifferentiation," Biochem. Biophys. Res. Commun. 353(3), 841847 (2007).

53. P. P. Provenzano, D. R. Inman, K. W. Eliceiri, and P. J. Keely, "Matrix density-induced mechanoregulation of breast cell phenotype, signaling and gene expression through a FAK-ERK linkage," Oncogene 28(49), 4326-4343 (2009).

54. G. Ranieri and G. Gasparini, "Angiogenesis and angiogenesis inhibitors: a new potential anticancer therapeutic strategy," Curr. Drug Targets Immune, Endocrine Metabolic Disorders 1(3), 241-53 (2001). 